\title{
Expression prosodique du focus et du donné au sein des groupes nominaux [N A] du français.
}

\author{
Fatima Hamlaoui, Sascha Coridun, Caroline Féry \\ Goethe-Universität Frankfurt Am Main \\ fham1044@gmail.com, saschacoridun@hotmail.de, caroline.fery@googlemail.com
}

\begin{abstract}
Dans cet article, nous rapportons les résultats d'une étude acoustique des corrélats prosodiques de l'expression de la structure de l'information au sein de groupes nominaux composés d'un nom et d'un adjectif. Cette recherche s'insère dans le cadre d'une étude en cours, et ne présente qu'une partie des résultats obtenus, ceux qui touchent le groupe nominal, l'expression du focus contrastif et du donné. Au moyen d'une tâche inspirée du «jeu d'animaux » du QUIS (Skopeteas et al. 2006, Swerts et al. 2002) et adaptée au français, nous avons testé l'hypothèse selon laquelle cette langue se distingue des langues germaniques en ce que le statut discursif du nom et de l'adjectif ne sont pas reflétés par la force accentuelle de ces éléments. Les résultats obtenus suggèrent que bien que les locuteurs produisent des indices acoustiques indiquant un degré de proéminence plus important, notamment du nom, lorsqu'il est focalisé que lorsqu'il est donné, ces indices sont moindres. Aussi, le français se distingue clairement des langues germaniques en ce qui concerne l'absence de désaccentuation post-focale au sein du groupe nominal.
\end{abstract}

\section{Introduction}

Malgré le fait qu'il ne s'agisse pas d'une tendance universelle, il a été montré que dans de nombreuses langues du monde, la structure informationnelle détermine au moins partiellement la réalisation prosodique des énoncés. ${ }^{1}$ Les langues font une utilisation différente des différents paramètres prosodiques (entre autres F0, durée, intensité). Dans les langues dans lesquelles la structure de l'information a une influence sur la prosodie, celle-ci peut se manifester de diverses façons: insertion/effacement de frontières prosodiques, changement de registres (dans les langues à tons par exemple), pitch accents/désaccentuation etc (entre autres, Bruce 1977 pour le suédois, Xu 1999 pour le chinois, Féry \& Ishihara 2010 pour l'allemand et le japonais ou encore Kanerva 1990 pour le chichewa).

Les études acoustiques antérieures portant sur la réalisation de séquences contenant des parties focalisées et des parties données en français ont montré que dans cette langue également, la structure de l'information a une influence sur la prosodie. Ainsi, il a été observé qu'aussi bien au niveau des phrases déclaratives simples qu'au niveau des phrases complexes (phrases clivées), les éléments donnés se trouvant en position post-focale se caractérisent par une intonation plate et relativement basse (entre autres, Delais et al. 2002, Jun \& Fougeron, 2000). Il a toutefois été observé qu'il existe une certaine variation dans la réalisation des éléments donnés. Jun \& Fougeron observent qu'en plus de ne pas présenter de différence systématique avec leur contrepartie non-donnée en ce qui concerne le paramètre de la durée, les séquences données en position post-focale présentent parfois des variations tonales.

Le français, et plus généralement les langues romanes, sont connus pour leur manque de plasticité prosodique (Vallduvi 1992). Dans les contextes où les langues germaniques ont recours à une stratégie purement prosodique pour mettre en valeur les constituants focalisés ou pour marquer des constituants comme donnés (Terken \& Hirschberg 1994 pour l'anglais ou encore Terken, 1984 pour le néerlandais),

\footnotetext{
${ }^{1}$ Remerciements : Cette recherche a été financée par la Fondation Alexander von Humboldt, à laquelle nous souhaitons exprimer ici toute notre gratitude. Nous souhaitons également remercier Annie Rialland, Laurent Roussarie et Gratianne Richer-Onate pour leur soutien.
} 
les langues romanes tendent à avoir recours à un changement dans l'ordre des mots (Lambrecht 1994). Diverses stratégies syntaxiques et morphologiques permettent soit de placer un élément focalisé dans une position dans laquelle un accent est réalisé par défaut (particulièrement la frontière droite des groupes prosodiques) soit d'ôter les éléments donnés d'une telle position (Hamlaoui 2009).

Le contexte syntaxique étudié ici présente un intérêt particulier en ce que, malgré le fait que la grammaire du français permette à certains adjectifs d'être produits aussi bien en position pré-nominale qu'en position post-nominale, aucun changement dans l'ordre des mots n'est possible en vue de satisfaire les exigences prosodiques liées à l'expression de la structure de l'information.

Dans leur étude comparative de l'italien et du néerlandais, Swerts et al. 2002 ont montré que la structure de l'information exerce une influence sur la réalisation prosodique du nom et de l'adjectif au sein des groupes nominaux ([A N]) du néerlandais mais non sur ceux des groupes nominaux ([N A]) de l'italien. Dans la première langue, les locuteurs ont recours à la désaccentuation pour indiquer le caractère donné des éléments. Ceci est particulièrement audible lorsque l'élément donné se trouve en position finale du groupe nominal, soit quand c'est le nom qui est donné. Les locuteurs italiens, eux, ont tendance à accentuer tous les mots, quel que soit leur statut sur le plan informationnel. Dans le cadre de cette étude, il a notamment été montré que les auditeurs italiens ne sont pas en mesure de reconstruire le contexte dans lequel un groupe nominal a été produit, à l'inverse des auditeurs néerlandais, qui obtiennent des scores relativement élevés dans cette tâche, particulièrement dans le cas des groupes nominaux dont la structure informationnelle est Focus-Donné.

Sur le plan phonologique, le français contraste avec les langues germaniques autant qu'avec d'autres langues romanes telles que l'italien et l'espagnol en ce qu'il ne présente pas d'accent lexical. Alors que ces langues tendent à associer les tons aux accents lexicaux (et réalisent ainsi ce qu'on nomme communément des pitch accents), le français tend à associer les tons aux extrémités des groupes prosodiques: généralement à la dernière syllabe du groupe, correspondant ainsi à l'accent primaire, et optionnellement à la première syllabe du groupe, l'accent secondaire. Malgré le fait que le français soit également une langue intonative, il se distingue des langues citées ci-dessus en ce qu'il présente un comportement de langue à groupement (phrase-based language) plutôt qu'un comportement de langue à proéminences (prominence-based language) (Féry, Pahaut, Hörnig 2010).

De quelle façon les locuteurs francophones encodent-ils la structure de l'information au sein des groupes nominaux ? Leur statut informationnel détermine-t-il le degré de proéminence de chacun des deux mots? Y a-t-il un changement dans le groupement de ces deux éléments ? L'hypothèse testée est la suivante : les notions de structure informationnelle prises en compte dans la présente étude n'ont pas d'effet significatif sur la proéminence du nom et de l'adjectif. En d'autres termes, nous faisons l'hypothèse que le français présente le même comportement que l'italien en ce qu'il n'a pas recours à la désaccentuation pour indiquer le statut donné d'un élément. De plus, en accord avec les résultats de Féry, Hörnig et Pahaut, nous postulons que le focus peut avoir une influence sur le groupement prosodique des éléments : un mot focalisé a tendance à former son propre syntagme prosodique ( $p$-phrase).

\section{Elicitation des groupes nominaux}

\subsection{Participants}

12 francophones natifs (10 femmes, 2 hommes) âgés de 18 à 47 ans et originaires d'Ile-de-France $(\mathrm{n}=9)$, de l'Oise $(n=1)$, du Poitou-Charentes $(n=1)$ et de Rhône-Alpes $(n=1)$, ont été recrutés dans le cadre universitaire et ont été payés pour leur participation.

\subsection{Design, matériel et procédure}

La méthode expérimentale utilisée dans la présente étude est inspirée de celle qui a été développée par Swerts et al. 2002 et reprise par Skopeteas et al. 2006. Assis à une table avec l'expérimentateur, les 
participants ont été priés de décrire une série d'images digitales représentant des animaux colorés (4 animaux x 4 couleurs) et ce «afin d'aider l'expérimentateur à classer la contrepartie papier de cette série d'images ». Avant de débuter l'expérience, plusieurs images ont été montrées aux participants de manière à les familiariser avec les animaux et les couleurs à produire.

Tous les mots cibles sont dissyllabiques et leurs consonnes sont majoritairement des consonnes sonantes. La liste de ces mots est donnée dans le Tableau 1.

Les images sont présentées une à une, sur l'écran d'un ordinateur, et ce dans le cadre d'une tâche individuelle. La série d'images est ordonnée de sorte à éliciter des groupes nominaux présentant trois configurations informationnelles distinctes : Focus-Donné (FD), Donné-Focus (DF) et Focus-Focus (FF). Le premier mot est toujours le nom, et le deuxième l'adjectif. Le Tableau 2 illustre les trois configurations informationnelles élicitées. On entend ici par mot «focalisé » un mot qui n'apparait pas dans le tour précédant. En dehors de la description du premier tour, tous les éléments focalisés sont en relation de contraste avec un des éléments du tour précédant. Un élément «donné» est quant à lui un élément qui figure dans la description du tour précédant.

Il a été demandé aux participants de produire uniquement la description de l'image et d'éviter tout autre mot. Les énoncés ont été enregistrés dans une pièce silencieuse, au moyen d'un magnétophone Edirol R$44(16 \mathrm{bits} / 44.1 \mathrm{kHz})$ et d'un micro-casque.

\begin{tabular}{|c|c|}
\hline Animaux & Couleurs \\
\hline Lama & Lilas \\
\hline Merlan & Marron \\
\hline Moineau & Orange \\
\hline Mulot & Violet \\
\hline
\end{tabular}

Tab 1. Mots cibles

\begin{tabular}{|c|c|c|}
\hline Tour & Image & Statut discursif \\
\hline 1 & Moineau marron & \\
\hline 2 & Lama marron & Focus Donné (FD) \\
\hline 3 & Lama orange & Donné Focus (DF) \\
\hline 4 & Mulot lilas & Focus Focus (FF) \\
\hline$\ldots$ & $\ldots$ & $\ldots$ \\
\hline
\end{tabular}

Tab.2. Illustration des trois statuts discursifs

\subsection{Traitement des données}

Les données audio ont été annotées et analysées sous PRAAT (Boersma \& Weenink 2011). Les valeurs suivantes ont été relevées semi-automatiquement à l'aide de scripts:

- Maximum et minimum F0 (en Hz) du nom et de l'adjectif et de chacune de leurs syllabes ; 
- Durée (en ms) du nom et de l'adjectif et de chacune de leurs syllabes ;

- Intensité moyenne (en dB) du nom et de l'adjectif et de chacune de leurs syllabes.

Nous avons également étudié la distribution et la durée des pauses internes aux groupes nominaux.

\section{Résultats}

Parmi les 624 énoncés produits par nos locuteurs, 19 soit (3\%) des données présentaient une erreur (le groupe nominal est produit au sein d'une phrase, une pause remplie sépare le nom et l'adjectif ou encore le locuteur produit une hésitation ou une reprise compromettant la prise de mesures). Ces énoncés ont été exclus de l'analyse. La plupart des énoncés analysés $(85 \%)$ présentent une montée de continuation ( $\mathrm{FF}=$ $157, \mathrm{DF}=180, \mathrm{FD}=176$, soit au total 513 stimuli), dans le cadre de ce que Swerts et al. appellent "une sorte d'intonation de liste", qui représente 50\% de leurs données du néerlandais. Swerts et al. (2002:642) observent un effet de la structure informationnelle sur l'intonation de leurs données du néerlandais aussi bien dans les énoncés à intonation finale descendante que dans ceux à intonation finale montante. Ce fait nous a encouragés à rechercher le même type de résultats dans les productions françaises. À cette fin, et en ce qui concerne la fréquence fondamentale, nous distinguons donc deux ensembles de données, que nous présentons séparément : tout d'abord les énoncés à intonation finale montante puis les énoncés à intonation finale descendante. Ces dernières étant en nombre réduit $(\mathrm{FF}=32, \mathrm{DF}=27, \mathrm{FD}=33)$ et n'influençant pas l'image générale obtenue, nous nous contentons de les résumer pour les paramètres de l'intensité et de la durée.

Nous avons appliqué le logiciel statistique R (R Core Development Team, 2011) pour ANOVA avec des facteurs fixes: Locuteur, Item et Structure Informationnelle/Focus. Les variables individuelles dépendantes pour le facteur Structure Informationnelle sont décrites ici.

\subsection{Fréquence fondamentale}

Les résultats présentés dans cette section ont été obtenus en normalisant les données de nos participants. La formule utilisée au sein de chaque groupe nominal est la suivante :

$\mathrm{F} 0$ normalisée $=\mathrm{F} 0$ absolue $-\mathrm{F} 0$ minimum $/ \mathrm{F} 0$ maximum $-\mathrm{F} 0$ minimum

En ce qui concerne les énoncés présentant une intonation montante, un léger effet du focus est observé sur la fréquence fondamentale du nom mais non sur celle de l'adjectif. La Figure 1 présente la fréquence fondamentale maximale moyenne au sein du nom et de l'adjectif pour chacune de nos trois conditions. 


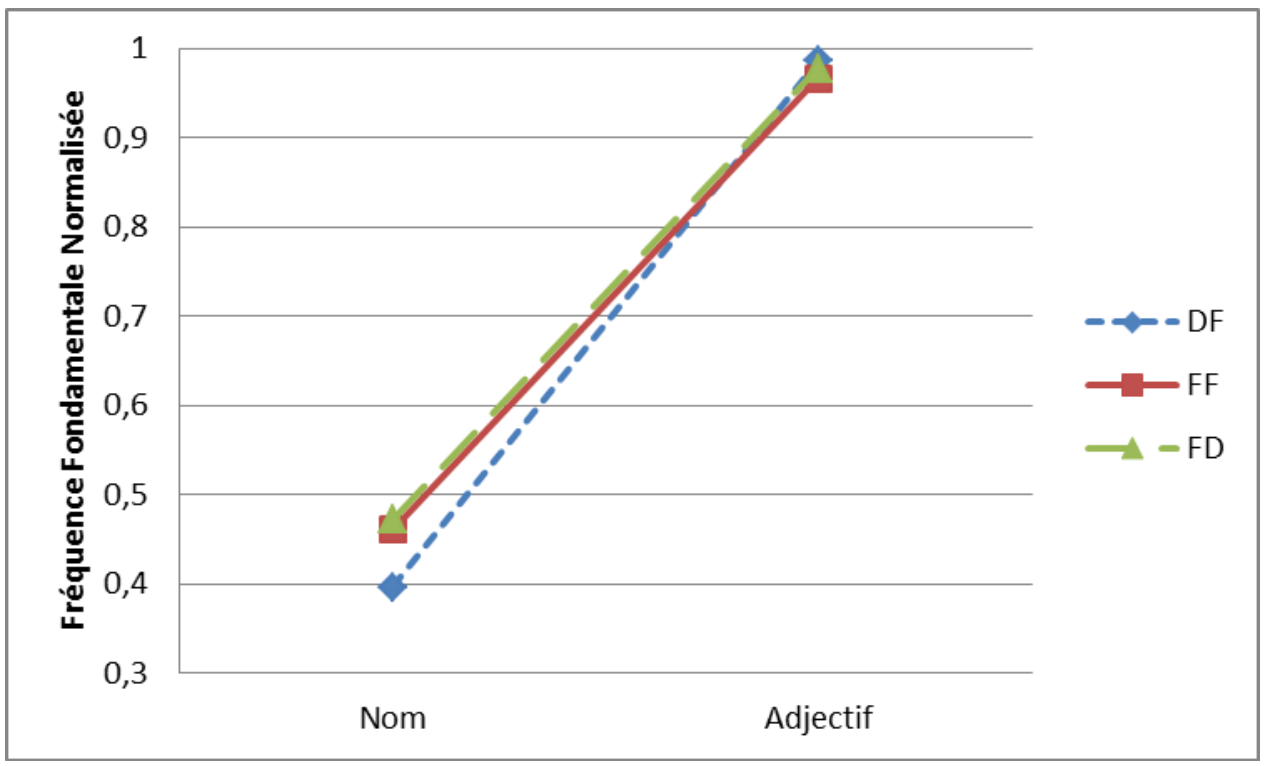

Fig. 1. Valeurs moyennes des maxima de F0 (normalisée) dans les conditions DF, FF et FF pour les énoncés à intonation montante

Nous n'observons pas d'effet significatif du facteur Focus sur la fréquence fondamentale maximale de l'adjectif $(F(2,485)=0.64$, ns. $))$. Néanmoins, la fréquence fondamentale du nom présente un effet significatif du focus $(F(2,485)=5,53 \mathrm{p}<0,005)$. Un test Post-Hoc montre que les noms dans la condition FD présentent une hauteur maximale moyenne plus importante que les noms dans la condition DF $(\mathrm{p}<0,01)$. Les noms de la condition FF présente également une hauteur maximale moyenne plus importante que les noms dans la condition DF $(\mathrm{p}<0,05)$. Il n'y a pas de différence significative entre la hauteur maximale moyenne des noms des conditions FF et FD.

Afin de rendre la comparaison possible avec les données de néerlandais de Swerts et al. présentant une intonation finale montante, nous avons également calculé la valeur de la différence de registre (en valeurs absolues) entre le nom et l'adjectif (excursion size of movement) : (Max F0 - Min F0) Nom $_{\text {- }}$ (Max F0 -

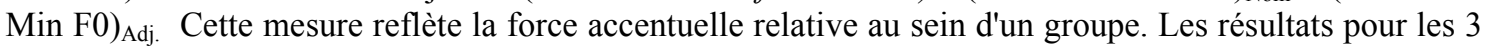
conditions sont représentés par la Figure 2. Les valeurs négatives s'expliquent par le fait que l'adjectif tend à présenter ici un registre plus haut que le nom, du fait de l'intonation finale montante. 


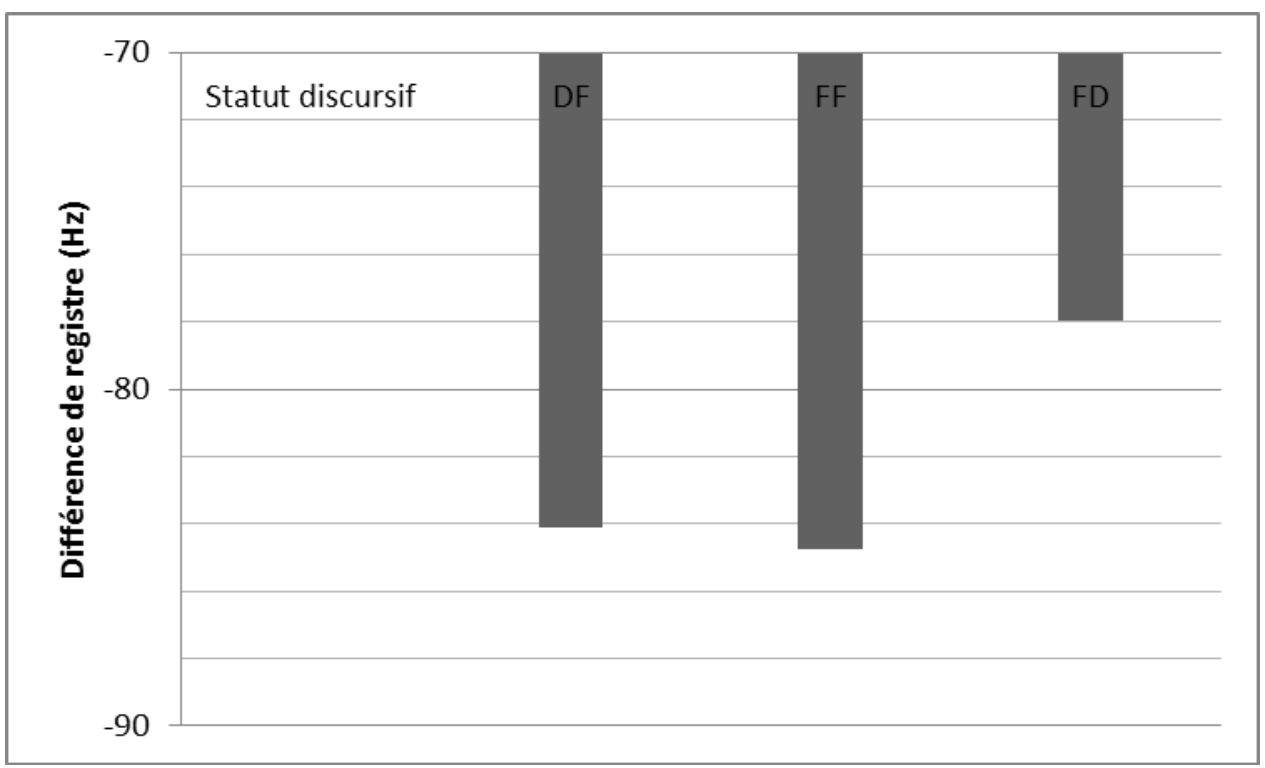

Fig. 2. Valeurs moyennes de la différence entre le registre du nom et de l'adjectif pour les conditions $\mathrm{DF}, \mathrm{FF}$ et $\mathrm{FD}$ des énoncés à intonation finale montante (uniquement les participantes féminines)

Les résultats montrent une légère influence du statut discursif sur la différence de registre du nom et de l'adjectif. Cette différence (non-significative) de registre entre le nom et l'adjectif est légèrement moindre lorsque le nom est focalisé, mais uniquement dans la condition FD et non dans la condition FF. Le français se distingue ici clairement du néerlandais. Dans les énoncés à intonation finale montante du néerlandais, la différence moyenne de registre entre l'adjectif et le nom dans la condition DF est négative (environ $-60 \mathrm{~Hz}$ ), ce qui indique que le second mot présente un registre plus important que le premier. Dans la condition FD, toujours en néerlandais, la différence de registre est positive (environ $20 \mathrm{~Hz}$ ) et le rapport est inversé : malgré l'intonation finale montante, le second mot présente un registre (reflétant la force accentuelle) moins important que le premier. Les résultats obtenus ici pour le français et présentés en Figures 1 et 2 indiquent que même si la force accentuelle du premier mot du groupe nominal est différente dans nos trois conditions, elle reste inférieure à celle du second mot qui, contrairement à ce qui se passe en néerlandais, n'est pas désaccentué lorsqu'il est donné.

Comme on peut le voir dans la Figure 3, au sein des énoncés présentant une intonation finale descendante, les noms focalisés ont tendance à être réalisés légèrement plus haut que les noms donnés. La même tendance est également visible sur l'adjectif. 


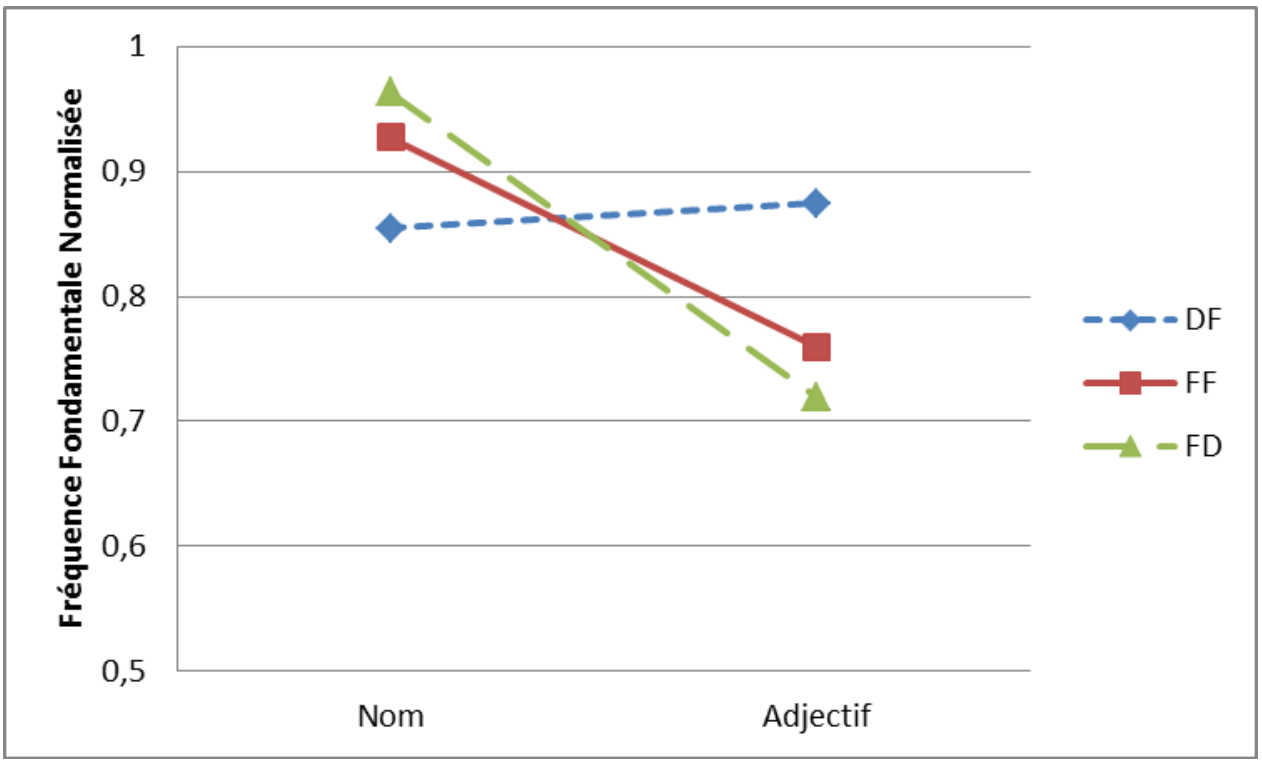

Fig. 3. Valeurs moyennes des maxima de F0 (normalisée) dans les conditions DF, FF et FF, énoncés à intonation descendante

La Figure 4, qui représente la différence de registre entre le nom et l'adjectif pour les énoncés à intonation descendante indique la même tendance que pour les énoncés à intonation montante : la différence de registre est réduite lorsque l'adjectif est focalisé et plus importante lorsque le nom est focalisé. Cela semble indiquer un effet de la structure informationnelle. Dans les énoncés à intonation descendante du néerlandais de Swerts et al., la différence moyenne de registre entre le premier mot et le second mot du groupe nominal est d'environ $10 \mathrm{~Hz}$ dans la condition DF et d'environ $50 \mathrm{~Hz}$ dans la condition FD. Nos données ne nous permettant pas pour le moment de déterminer si la différence de hauteur entre l'adjectif dans les conditions FD et DF est significative, davantage de recherches sont actuellement en cours. Néanmoins, les valeurs présentées dans la figure 4 montrent une différence de registre atteignant maximalement $18 \mathrm{~Hz}$. Nous sommes donc loin des $50 \mathrm{~Hz}$ obtenus pour le néerlandais. 


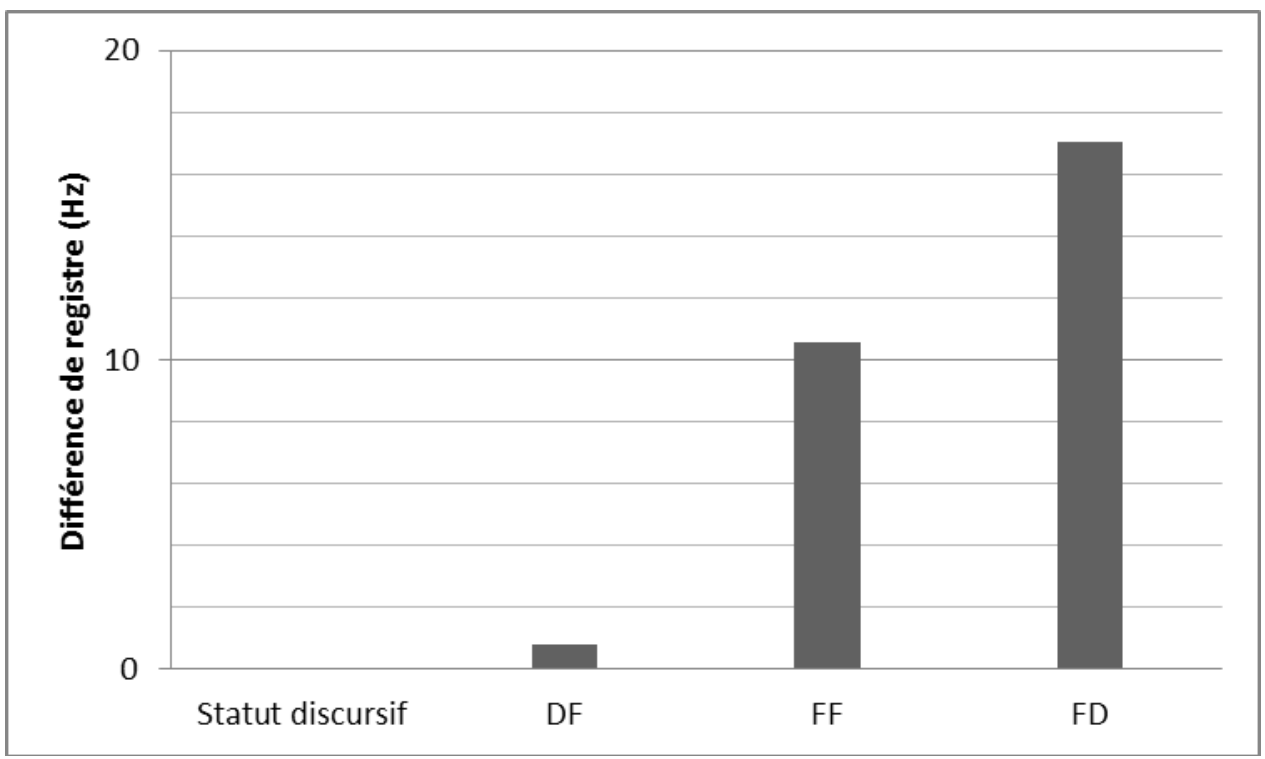

Fig. 4. Valeurs moyennes de la différence entre le registre du nom et de l'adjectif pour les conditions DF, $\mathrm{FF}$ et $\mathrm{FD}$ des énoncés à intonation finale descendante (uniquement les participantes féminines).

En résumé, en ce qui concerne l'effet du focus et du donné sur la fréquence fondamentale au sein du groupe nominal, nous avons observé une tendance des éléments focalisés à être réalisés plus haut que les éléments donnés correspondants. Cet effet s'est révélé être statistiquement significatif uniquement en ce qui concerne le nom des énoncés à intonation montante. La réalisation de l'adjectif semble, elle, rester stable dans les trois conditions. Au moment de l'écriture de cet article davantage de recherches sont en cours concernant les énoncés à intonation descendante.

\subsection{Durée}

Les résultats présentés dans cette section ont été obtenus en normalisant les données de nos participants.

Pour chaque groupe nominal et chaque participant, nous avons appliqué la formule suivante :

Durée transformée $=$ durée absolue de $\mathrm{x} /$ durée moyenne de $\mathrm{x}_{1} \ldots \mathrm{x}_{\mathrm{n}}$

Les moyennes des mesures de durée (normalisée) du nom et de l'adjectif pour les 3 conditions sont présentées dans la Figure 5 pour les énoncés présentant une intonation montante. Des valeurs d'écart-type élevées indiquent une importante variation au sein des données collectées. 


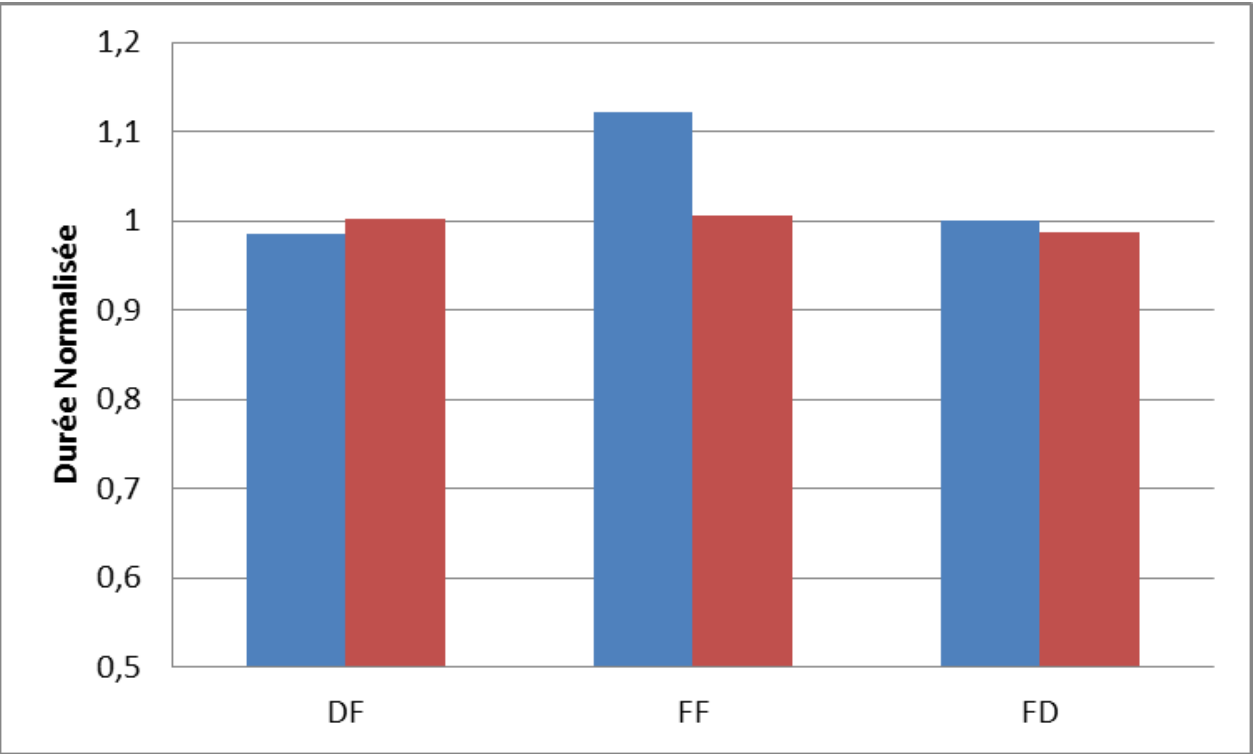

Fig. 5. Durée moyenne du nom et de l'adjectif dans les conditions DF, FF et FD pour les énoncés à intonation montante

Nous avons observé un effet significatif du focus sur la durée du nom $(F(2,485)=11,22, \mathrm{p}<0,01)$. Un test Post-Hoc indique que la différence de durée du nom dans les conditions DF et FF d'une part, et dans les conditions FF et FD d'autre part, est statistiquement significative ( $<<0,001$ dans les deux cas). La durée de l'adjectif ne présente, quant à elle, pas de différence significative sous l'effet du focus $(F(2,485, \mathrm{~ns}))$.

Les durées respectives du nom et de l'adjectif dans les énoncés à intonation descendante sont similaires aux résultats présentés ici. L'effet de finalité est légèrement moindre dans la condition $\mathrm{FF}$, ce qui ne change pas l'image générale obtenue, qui se résume ainsi : le mot final est plus long que le mot initial et le nom tend à être plus long lorsqu'il est focalisé que lorsqu'il est donné.

\subsection{Intensité}

L'intensité moyenne du nom et de l'adjectif dans les groupes nominaux à intonation montante est donnée en Figure 7 pour les 3 conditions. On observe que l'intensité va décroissante: dans les 3 conditions, le nom présente une intensité moyenne plus importante que celle de l'adjectif.

Une tendance similaire est observée en ce qui concerne les énoncés à intonation descendante. Nous nous limitons ici à la présentation des énoncés à intonation montante afin de présenter un groupe de données homogène. 


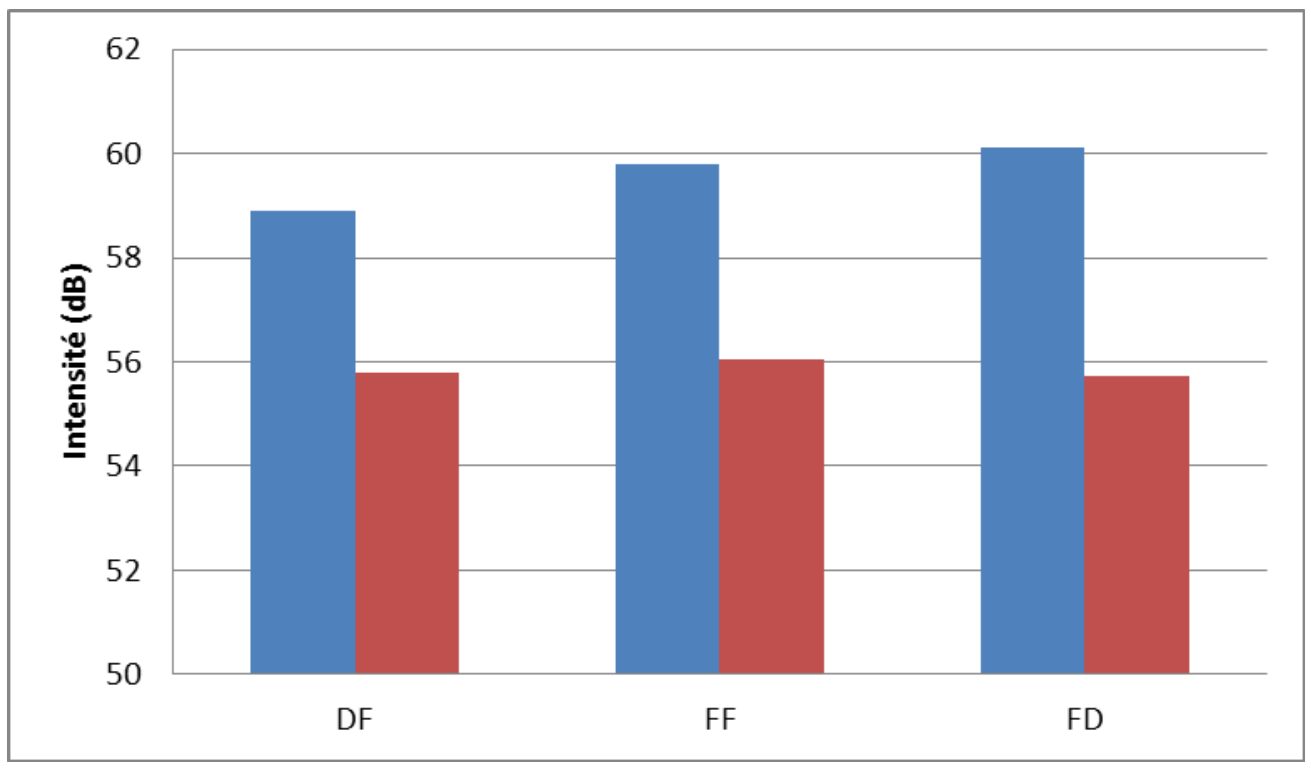

Figure. 6. Intensité moyenne du nom et de l'adjectif dans les conditions DF, FF et FD, pour les énoncés à intonation montante

Nous observons un effet significatif du facteur Focus sur l'intensité du nom $(F(2,483)=16,76$, $\mathrm{p}<0,0001)$. De plus, l'intensité de la première syllabe du nom présente un effet significatif du Focus $(F(2$, $485)=15.91, \mathrm{p}<0.0001)$, ainsi que la deuxième syllabe du nom $(F(2,485)=13,67, \mathrm{p}<0.0001))$. Un test Post-Hoc indique que l'intensité moyenne du nom est différente entre les conditions DF et $\mathrm{FF}(\mathrm{p}<0,001)$ ainsi qu'entre les conditions $\mathrm{FD}$ et $\mathrm{DF}(\mathrm{p}<0001)$. En ce qui concerne l'adjectif, seule la première syllabe présente un effet significative du facteur Focus $(F(2,485)=3,73, \mathrm{p}<0,05)$. Un test Post-Hoc montre que l'intensité moyenne de la première syllabe de l'adjectif se distingue uniquement dans les conditions $\mathrm{FF}$ et DF $(p<0,05)$. L'intensité est plus importante dans la condition FF que dans la condition DF. L'intensité de l'adjectif semble donc dépendre de celle du nom. Quand le nom est prononcé avec plus d'intensité pour des raisons discursives, l'adjectif fait de même pour atteindre un seuil comparable de proéminence. Si l'adjectif est le seul élément focalisé, le nom a moins d'intensité, et l'adjectif est également moins intense.

En résumé, le nom présente une intensité légèrement plus forte lorsqu'il est focalisé que lorsqu'il est donné. L'intensité de l'adjectif, elle, est plus variable, comme nous venons de le décrire.

\subsection{Pauses internes}

La distribution des pauses (inférieures à $500 \mathrm{~ms}$ ) et leur durée moyenne (en ms) pour chaque condition sont données au Tableau 3. Nous ne pouvons exclure ici la possibilité qu'un certain nombre de pauses d'hésitation soient prises en compte dans ces chiffres. Ces pauses sont en général nettement plus longues que les pauses grammaticales. En choisissant d'éliminer du tableau les pauses plus longues que $500 \mathrm{~ms}$, nous ne prenons pas en compte les pauses d'hésitation les plus importantes. 


\begin{tabular}{|c|c|c|}
\hline $\begin{array}{c}\text { Statut } \\
\text { discursif }\end{array}$ & Distribution & Durée moyenne \\
\hline DF & $55 / 213$ & 104,3 \\
& $25,8 \%$ & $(71,5)$ \\
\hline FF & $58 / 185$ & 152,7 \\
& $31,3 \%$ & $(113,3)$ \\
\hline FD & $47 / 208$ & 115 \\
& $22,6 \%$ & $(123,8)$ \\
\hline
\end{tabular}

Tab. 3. Distribution et durée moyenne (en ms) des pauses internes au groupe nominal (tous types d'énoncés confondus).

Dans les données que nous avons collectées, les pauses sont plus fréquentes et tendent à être plus longues dans la condition FF que dans les deux autres conditions. Les pauses tendent également à être légèrement plus longues dans la condition FD, que dans la condition DF.

\section{Etude de perception}

L'analyse acoustique des données recueillies montre que la position du focus exerce un effet significatif sur la F0, la durée et l'intensité du nom, mais pas de l'adjectif. Il s'agit de savoir si cette différence est suffisante pour permettre aux participants d'identifier la structure informationnelle du groupe nominal.

Le français est une langue à groupements prosodiques dans laquelle ces groupements peuvent également être encodés par des indices autres que ceux que nous avons mesurés. A titre d'illustration, diverses études ont montré que l'articulation des segments pouvait être influencée par leur position au sein des groupes prosodiques (entre autres, Fougeron \& Keating 1997, Fougeron 2001 et Féry 2004). Afin de prolonger cette étude et de déterminer si les auditeurs francophones sont en mesure d'identifier la structure informationnelle des différents groupes nominaux collectés au cours de notre expérience de production, nous avons mené un test de perception dont nous présentons ici les premiers résultats.

\subsection{Participants et design}

Au moment de l'écriture de cet article, 13 francophones natifs ( 12 femmes, 1 homme) âgés de 20 à 51 ans et originaires d'Ile-de-France $(\mathrm{n}=10)$, de la région Centre $(\mathrm{n}=1)$ et de la région Rhône-Alpes $(\mathrm{n}=1)$ ont pris part à cette expérience. Ils ont tous été recrutés dans le cadre universitaire.

L'expérience de perception a été élaborée à l'aide du logiciel PRAAT. 5 listes comportant un total de $2 \mathrm{x}$ 48 stimuli +4 stimuli d'entraînement ont été créées. Chaque liste contient 2 répétitions d'un même stimulus, et nous avons veillé à ce que chacun des stimuli contenus dans ces 5 listes proviennent d'un locuteur différent de manière à éviter tout effet lié à un locuteur particulier. Les stimuli ont été présentés aux participants sur ordinateur, sous la forme de 4 blocs de 25 stimuli, séparés par une pause dont la longueur a été fixée librement par les participants. Les participants ont d'abord été informés du cadre dans lequel les groupes nominaux qu'ils allaient entendre avaient été produits, ainsi que l'objectif recherché par les expérimentateurs : déterminer si la réalisation prosodique d'un groupe nominal est influencée par 
le groupe nominal qui le précédait. La tâche consistait à entendre un groupe nominal et à choisir le contexte dans lequel il avait été produit, tel que brièvement illustré au Tableau 4.

\begin{tabular}{|c|c|c|}
\hline Stimulus & Bouton de gauche & Bouton de droite \\
\hline Mulot violet & Mulot orange & Moineau violet \\
\hline Lama marron & Merlan marron & Mulot orange \\
\hline Merlan lilas & Lama violet & Merlan orange \\
\hline$\ldots$ & $\ldots$ & $\ldots$ \\
\hline
\end{tabular}

Tab. 4. Exemple de suite de stimuli utilisée dans le test de perception

Pour chaque stimulus entendu, les participants avaient le choix entre deux groupes nominaux: l'un constituant le contexte approprié et l'autre un contexte inapproprié. Nous avons veillé à ce que les différentes conditions soient également distribuées aussi bien pour les réponses appropriées que pour les réponses inappropriées. Nous nous sommes également assurés que chaque combinaison de nos 3 conditions en tant que réponse appropriée-réponse inappropriée (FD vs FF, FF vs FD, FF vs DF, DF vs FF etc), soit égale dans chacune des listes. Enfin, nous avons également veillé autant que possible à une distribution équitable des conditions et des réponses correctes et incorrectes entre les boutons de gauche et de droite.

\subsection{Résultats}

Dans leur étude, Swerts et al. observent que les locuteurs de néerlandais obtiennent un taux de réussite élevé dans une tâche consistant à identifier le contexte dans lequel un groupe nominal entendu en isolation avait été produit. Pour les mots focalisés, les participants néerlandais ont émis des jugements corrects oscillants entre 61 et $85 \%$. Ce résultat venait corroborer ceux de leur analyse acoustique, qui montraient que, dans cette langue, la force accentuelle relative au sein du groupe nominale était fonction de la structure informationnelle de ce groupe nominal.

Nos participants obtiennent quant à eux des résultats relativement faibles. Derrière un taux de réussite à première vue médiocre de reconnaissance des groupes nominaux produits dans les conditions FD (63\%) et proche du seuil critique $(50 \%)$ pour DF $(59 \%)$ et faible pour FF $(26 \%)$ se cachent diverses stratégies qui laissent penser que leurs choix ne reposent probablement que rarement sur les indices prosodiques du stimulus entendu. Ainsi le taux de réussite est particulièrement élevé pour les conditions FD et DF lorsque la réponse incorrecte correspond à la condition FF. En d'autres termes, les participants semblent préférer les réponses qui présentent un mot similaire au stimulus entendu au détriment des réponses dont les deux mots sont différents de ceux du stimulus entendu. Le taux de réussite s'élève à $69 \%$ pour la condition DF lorsque la réponse incorrecte correspond à la condition FF et seulement à $49 \%$ lorsque la réponse incorrecte correspond à la condition DF. Lorsqu'il s'agit d'identifier les groupes nominaux de la condition $\mathrm{FD}$, leur taux de réussite s'élève à $70 \%$ lorsque la réponse incorrecte correspond à la condition $\mathrm{FF}$, mais seulement à $57 \%$ lorsque la réponse incorrecte correspond à la condition $\mathrm{DF}$.

En résumé, les différences de durée, de hauteur et d'intensité mesurées dans le cadre de notre étude acoustique ne semblent pas indiquer à nos auditeurs la structure informationnelle des différents groupes nominaux collectés. Il nous paraît que si les indices avaient été suffisants pour identifier la structure informationnelle des différents groupes nominaux, le type de réponse incorrecte n'aurait pas joué un tel rôle dans nos résultats. 


\section{Discussion et perspectives}

L'étude que nous avons rapportée ici est adaptée d'une expérience de Swerts et al. 2002. Cette expérience a permis d'éliciter des groupes nominaux dans trois conditions : focus sur le nom et l'adjectif, focus uniquement sur le nom et focus uniquement sur l'adjectif.

Dans l'ensemble de données présentant une intonation finale montante, qui constitue $85 \%$ de nos données, nous avons observé une légère différence de production entre les mots focalisés et les mots donnés en ce qui concerne la fréquence fondamentale et l'intensité. Cet effet s'est révélé être statistiquement significatif uniquement en ce qui concerne le nom. Ainsi, le nom focalisé (dans les conditions FF et FD) tend à présenter une fréquence fondamentale plus haute et une intensité plus forte que sa contrepartie donnée (dans la condition DF). Nous avons également observé des différences de durée du nom entre les trois conditions. Cet effet ne semble cependant pas corréler avec la position du focus, puisque le nom tend uniquement à être plus long dans la condition FF et non dans la condition FD. Pour ce qui est de la fréquence fondamentale, la durée et l'intensité de l'adjectif, aucune différence statistiquement significative n'a été observée entre les trois conditions.

Dans l'ensemble de nos données, nous avons enfin observé que les pauses étaient légèrement plus fréquentes dans la condition FF que dans les deux autres conditions et qu'elles étaient, en moyenne, plus longues dans les conditions FF et FD que dans la condition DF. Ceci pourrait suggérer une tendance à insérer une frontière de syntagme prosodique après l'élément focalisé.

En néerlandais comme en allemand ou en anglais, le type de focus élicité ici, un focus contrastif, est accompagné d'une augmentation sensible de la fréquence fondamentale ainsi que d'une compression postfocale, sous la forme d'une compression du registre de l'élément qui suit le focus. Dans ces langues, les deux corrélats semblent jouer un rôle d'importance dans la perception de la structure informationnelle du groupe nominal. Clairement, ce n'est pas ce que nous avons observé en français. Nous avons mesuré une différence de fréquence fondamentale entre les éléments focalisés et les éléments donnés mais aucun indice de compression post-focale telle qu'observée dans les langues germaniques.

Le français se distingue également de l'italien. Swerts et al. n'avaient pu constater de différence entre les différentes conditions dans cette langue. En français, nous trouvons quelques différences de fréquence fondamentale et d'intensité. Nous observons néanmoins que les auditeurs francophones présentent un comportement similaire à celui des auditeurs italiens et ne sont pas en mesure de reconstruire le contexte dans lequel un groupe nominal a été produit. Le test de perception mené jusqu'ici ne permettant pas de tirer de conclusions définitives, et une nouvelle étude de perception est en cours.

L'hypothèse formulée au début de cet article selon laquelle le français se distingue des langues germaniques en ce que le statut discursif du nom et de l'adjectif n'est pas reflété par des différences de force accentuelle se trouve confirmée. Une autre hypothèse selon laquelle l'élément focalisé formerait une phrase prosodique doit être suspendue. Le groupe nominal ne peut être séparé en deux phrases prosodiques vu la cohésion syntaxique des deux mots utilisés dans notre expérience. Des phrases plus longues divisées en éléments focalisés et donnés nous semblent plus propices à fournir des indications quant à l'hypothèse de phrasage prosodique. Seules des analyses d'études empiriques plus approfondies permettront de vérifier ou de rejeter les hypothèses avancées dans cet article, une tâche à laquelle nous continuerons de nous consacrer dans les mois qui viennent. 


\section{Références bibliographiques}

Boersma, P. et Weenink, D. (2003-2012). PRAAT: Doing Phonetics by Computer.

Bruce, G. (1977) Swedish word accent in sentence perspective. Travaux de l'Institut de Linguistique de Lund 12 , Lund : Gleerup.

Delais-Roussarie, E., Rialland, A., Doetjes, J. et Marandin , JM. (2002). The Prosody of post-focus sequences in French. In Bel, G et Marlien, I. (éditeurs), Proceedings SPEECH PROSODY 2002. Aix-en-Provence.

Féry, C., Hörnig, R. et Pahaut, S. (2010). Phrasing in French and German: an experiment with semi-spontaneous speech. Dans : Gabriel, C. et Lleó, C. (éditeurs.) Intonational Phrasing at the Interfaces: Cross-Linguistic and Bilingual Studies in Romance and Germanic. Amsterdam : Benjamins, 11-41

Féry, C. et Ishihara S. (2010). How Focus and Givenness Shape Prosody. In Zimmermann, M. \& C. Féry (Eds.) Information Structure. Theoretical, Typological, and Experimental Perspectives. Oxford. Oxford University Press. 36-63.

Féry, C. (2004). Gradient prosodic correlates of phrasing in French. Dans : Meisenburg, T. et Selig, M. (éditeurs) Nouveaux Départs en Phonologie. Tübingen : Gunter Narr Verlag.

Fougeron C. (2001). Articulatory properties of initial segments in several prosodic constituents in French. Journal of Phonetics, 29(2), 109-135.

Fougeron C. et Keating P. (1997). Articulatory strengthening at edges of prosodic domain. Journal of the Acoustical Society of America, 106 (6), 3728-3740.

Hamlaoui, F. (2009). Le focus à l'interface de la syntaxe et de la phonologie : le cas du français dans une perspective typologique. Thèse de Doctorat. Université Paris III.

Jun, S.-A. et Fougeron, C. (2000). A phonological model of French intonation. Dans : Botinis, A. (éditeur) Intonation : Analysis, Modeling and Technology. Dordrecht : Kluwer Academic Publishing, 209-242.

Jun S.A. \& Fougeron C. (2002). The Realizations of the Accentual Phrase in French Intonation, Probus, 14, 147-172, A special issue on Intonation in the Romance Languages.

Kanerva, Jonni M. (1990) Focusing on Phonological Phrases in Chichewa. In: Inkelas, Sharon \& Draga Zec (eds.) Phonology-Syntax-Interface. Chicago: University of Chicago Press, 145-161.

Lambrecht, K. (1994). Information structure an sentence form: Topic, focus, and the mental representation of discourse referents. Cambridge, Massachusetts : Cambridge University Press.

R Development Core Team (2011). R: A language and environment for statistical computing. R Foundation for Statistical Computing, Vienna, Austria. ISBN 3-900051-07-0, URL http://www.R-project.org/.

Skopeteas, S., Fiedler, I., Hellmuth, S., Schwarz, A., Stoel, R., Fanselow, G., Féry, C., and Krifka, M. (2006). Questionnaire on information structure (QUIS): Reference manual. Interdisciplinary Studies on Information Structure.

Swerts, M., Krahmer, E., et Avesani, C. (2002). Prosodic marking of information status in Dutch and Italian: A comparative analysis. Journal of Phonetics, 30, 629-654.

Terken, J. (1984). The Distribution of Accents in Instructions as a Function of Discourse Structure, Language and Speech, 27, 269-289.

J. Terken and J. Hirschberg (1994) Deaccentuation and Persistence of Grammatical Function and Surface Position. Language and Speech, 37(2), 125-145.

Vallduví, E. (1992). The Informational Component. New York : Garland.

$\mathrm{Xu}, \mathrm{Y}$. (1999). Effects of tone and focus on the formation and alignment of F0 contours. Journal of Phonetics, 27, 55105. 